\title{
Non-tuberculous mycobacteria: epidemiological pattern in a reference laboratory and risk factors associated with pulmonary disease
}

\author{
J. MENCARINI ${ }^{1}$, C. CRESCI ${ }^{2}$, M. T. SIMONETTI ${ }^{3}$, C. TRUPPA ${ }^{1}$, \\ G. CAMICIOTTOLI ${ }^{2,4}$, M. L. FRILLI ${ }^{4}$, P. G. ROGASI ${ }^{5}$, S. VELOCI ${ }^{1}$, \\ M. PISTOLESI $I^{2,4}$, G. M. ROSSOLINI ${ }^{3,6,7}$, A. BARTOLONI ${ }^{1,5}$ AND \\ F. BARTALESI ${ }^{5 *}$ \\ ${ }^{1}$ Infectious Diseases Unit, Department of Experimental and Clinical Medicine, University of Florence, Florence, \\ Italy \\ ${ }^{2}$ Pneumology Unit, Careggi Hospital, Florence, Italy \\ ${ }^{3}$ Tuscany Regional Reference Centre for Mycobacteria, Microbiology and Virology Unit, Careggi Hospital, \\ Florence, Italy \\ ${ }^{4}$ Section of Respiratory Medicine, Department of Experimental and Clinical Medicine, University of Florence, \\ Florence, Italy \\ ${ }^{5}$ Unit of Infectious and Tropical Diseases, Careggi Hospital, Florence, Italy \\ ${ }^{6}$ Section of Microbiology, Department of Experimental and Clinical Medicine, University of Florence, Florence, \\ Italy \\ ${ }^{7}$ Department of Medical Biotechnologies, University of Siena, Siena, Italy
}

Received 8 April 2016; Final revision 7 October 2016; Accepted 9 October 2016;

first published online 2 November 2016

\section{SUMMARY}

The diseases caused by non-tuberculous mycobacteria (NTM), in both AIDS and non-AIDS populations, are increasingly recognized worldwide. Although the American Thoracic Society published the guidelines for diagnosis of NTM pulmonary disease (NTM-PD), the diagnosis is still difficult. In the first part of the study, we collected data on NTM isolates in the Mycobacteriology Laboratory of Careggi Hospital (Florence, Italy) and analysed the epidemiological data of NTM isolates. Then, to analyse the risk factors associated to NTM-PD, we studied the presence of ATS/IDSA criteria for NTM-PD in patients who had at least one positive respiratory sample for NTM and were admitted to the Infectious Disease Unit and the Section of Respiratory Medicine. We selected 88 patients with available full clinical data and, according to ATS/IDSA criteria, classified 15 patients (17\%) as NTM-PD cases and 73 as colonized patients $(83 \%)$. When comparing colonized and NTM-PD patients we did not find significant differences of age, gender and comorbidity. We observed that Mycobacterium avium and $M$. intracellulare were statistically associated with NTM-PD $(P=0 \cdot 001)$ whereas $M$. xenopi was statistically associated with colonization. Although the number of studied patients is limited, our study did not identify risk factors for NTM-PD that could help clinicians to discriminate between colonization and disease. We underline the need of close monitoring of NTM-infected patients until the diagnosis is reasonably excluded. Further larger prospective studies and new biological markers are needed to identify new useful tools for the diagnosis of NTM-PD.

Key words: Mycobacteria, respiratory infections.

\footnotetext{
* Author for correspondence: Dr F. Bartalesi, Infectious and Tropical Disease Unit, Careggi Hospital, Largo Brambilla 3, 50134, Florence, Italy.

(Email: bartalesif@aou-careggi.toscana.it)
} 


\section{INTRODUCTION}

Non-tuberculous mycobacteria (NTM) are environmental organisms residing in soil and water, with low pathogenicity to humans. Over recent decades, the isolation of NTM, and the disease caused by NTM in both AIDS and non-AIDS populations have been increasingly reported worldwide [1-3]. This rise is certainly due to the increase in number and quality of mycobacteriology laboratories, and the improvement of laboratory techniques, as well as to the advent of AIDS in the 1980s. However, there are probably other factors that can justify this trend, such as the larger number of immunocompromised patients surviving today, rising life expectancy and the availability of computed tomography scanning $[4,5]$.

NTM disease and its pattern of presentation are related to the interactions between species-specific virulence factors and host factors. Although generally NTM virulence is low, it may differ between species and some strains might demonstrate different virulence [6-8]. Regarding the host, pre-existing structural lung disease [tuberculosis, cystic fibrosis, chronic obstructive pulmonary disease (COPD), silicosis and other pneumoconiosis], alcoholism, smoking, oesophageal reflux and alterations of innate or acquired immunity are acknowledged risk factors for development of NTM pulmonary disease (NTM-PD) [9, 10].

The NTM infection's clinical presentation is nonspecific and in the clinical setting it is not easy to distinguish environmental contamination with transient colonization from pulmonary disease. In order to standardize the definition for NTM-PD and to reach a more accurate diagnosis, in 2007 the American Thoracic Society, in collaboration with the Infectious Disease Society of America (ATS/IDSA), published guidelines for the diagnosis, treatment and prevention of NTM disease. In this guideline, it is specified that the diagnosis of NTM-PD requires the presence of respiratory symptoms, radiological features, microbiological criteria and the exclusion of other diagnoses [11].

The clinical relevance of NTM species could differ by region or setting and some authors suggest modulation of diagnostic criteria according to clinical relevance of each species $[9,12]$.

Considering the complexity of the NTM-PD diagnosis, due to the difficulty in applying ATS/IDSA criteria in different settings, we collected data on NTM isolates in the mycobacteriology laboratory of our hospital over 2 years, with the aim of contributing to clarifying the application of those criteria in clinical practice and to describing our local epidemiology. We identified patients with positive specimens from the Infectious Disease Unit and the Section of Respiratory Medicine and collected their medical records to classify the patients by the presence of the ATS/IDSA criteria for NTM-PD.

\section{MATERIALS AND METHODS}

\section{Data collection}

We retrospectively reviewed the database of the mycobacteria isolated in a mycobacteriology laboratory (Tuscany Regional Reference Centre for Mycobacteria, Careggi Hospital, Florence, Italy) for the period from January 2011 to December 2012. We excluded 349 mycobacteria of the Mycobacterium tuberculosis complex and 32 NTM isolated from non-respiratory specimens. Finally, we identified a total of 554 NTM isolated from respiratory samples [sputum, bronchoalveolar lavage (BAL), pleural effusion]. We analysed the epidemiological data of NTM isolates, considering also the demographic characteristic (age, sex, geographical origin) of the patients with positive specimens. In the second part of our study, among the patients with NTM isolates, we selected those admitted to the Infectious Disease Unit and the Section of Respiratory Medicine who had full clinical data available. For each patient we collected clinical and radiological information from clinical records. We define a 'NTM-PD case' as a patient fulfilling ATS/IDSA diagnostic criteria (clinical, microbiological and radiological criteria) in the absence of other pulmonary infections. We compared clinical data between patients defined as NTM-PD cases and as NTM-PD colonized, to identify potential risk factors associated with NTM-PD.

\section{Laboratory diagnosis}

Specimens were decontaminated using a standard $\mathrm{N}$ acetyl-L-cysteine- $\mathrm{NaOH}$ method. A rhodamine-auramine stain method was utilized to analyse specimens for acidfast bacilli. Cultures were performed on liquid and solid medium at $35-37^{\circ} \mathrm{C}$. As liquid medium, a nonradiometric automated system $\left(\mathrm{BATECT}^{\mathrm{TM}} \mathrm{MGIT}^{\mathrm{TM}}\right.$ 960, Becton Dickinson, USA) was used to detect mycobacterial growth; solid media (Lowenstein-Jensen) were observed weekly for up to 2 months. Positive cultures were microscopically confirmed and identified to differentiate $M$. tuberculosis complex from NTM using a rapid 
immunochromatographic test to detect MTbc-specific MPT64 antigen (MGIT TB identification test). A further differentiation up to species level was performed using commercially available molecular genetic methods (line probe assay): Hain GenoType ${ }^{\circledR}$ MTBC for differentiation of the M. tuberculosis complex and Hain GenoType ${ }^{\mathbb{R}}$ Mycobacterium CM (Hain Lifescience $\mathrm{GmbH}$, Germany) for non-tuberculous more common mycobacteria. Another Hain kit was used to identify some uncommon species (GenoType ${ }^{\circledR}$ Mycobacterium AS, Hain Lifescience $\mathrm{GmbH}$ ) [13]. In some cases a further sequencing of specific genetic targets was needed in order to differentiate species between a complex (e.g. M. avium complex) or a group (e.g. M. fortuitum group) or to detect some infrequently encountered species [14]. The obtained sequences were compared with the GenBank database in order to match the best species identity.

\section{Statistical analysis}

Data were analysed using Stata software v. 11.0 (StataCorp, USA). The statistical significance of the differences in quantitative data between the two groups (NTM colonized patients and NTM-PD cases) was determined using the Wilcoxon rank sum test. Pearson's $\chi^{2}$ test was used for statistical correlations between qualitative variable, applying Fisher's exact test when needed. Differences were considered significant for $P$ values $\leqslant 0 \cdot 05$.

\section{Ethical standards}

The ethics committee of Careggi Hospital approved the study (register no. 57/13). The authors assert that all procedures contributing to this work comply with the ethical standards of the relevant national and institutional committees on human experimentation and with the Helsinki Declaration of 1975, as revised in 2008.

\section{RESULTS}

\section{Epidemiological data}

A total of 554 NTM were isolated from 462 patients, between January 2011 and December 2012. Most $(66 \%)$ patients were male, with a mean and median age of 64 and 69 years, respectively. The majority of patients came from Europe (413, 89.4\%), particularly from Italy $(393,85 \cdot 1 \%)$. The majority of isolates were from sputum specimens $(372,67 \cdot 1 \%)$. Three hundred and ninety-one patients $(84.6 \%)$ had only one species of NTM isolated from one source, $29(6 \cdot 3 \%)$ had
Table 1. Microbiological date of the 554 NTM isolated from respiratory sample in the years 2011-2012

\begin{tabular}{ll}
\hline \hline Variable & Samples, $n(\%)$ \\
\hline Isolation sources & \\
Sputum & $372(67 \cdot 1)$ \\
Bronchoalveolar lavage & $113(20 \cdot 5)$ \\
Bronchial aspirated & $66(11 \cdot 9)$ \\
Pleural fluid & $3(0 \cdot 5)$ \\
Species identification & \\
Mycobacterium xenopi & $219(39 \cdot 5)$ \\
Mycobacterium avium complex & $216(38 \cdot 9)$ \\
M. chimaera & $100(46 \cdot 3)$ \\
M. avium & $78(36 \cdot 1)$ \\
M. intracellulare & $37(17 \cdot 1)$ \\
M. bouchedurhonense & $1(0 \cdot 5)$ \\
Rapidly growing mycobacteria & $39(7)$ \\
M. abscessus & $22(56 \cdot 4)$ \\
M. fortuitum & $8(20 \cdot 5)$ \\
M. chelonae & $3(7 \cdot 7)$ \\
M. massiliense & $3(7 \cdot 7)$ \\
M. mucogenicum & $2(5 \cdot 1)$ \\
M. alvei & $1(2 \cdot 6)$ \\
Mycobacterium gordonae & $52(9 \cdot 4)$ \\
Mycobacterium arupense & $7(1 \cdot 3)$ \\
Mycobacterium lentiflavum & $6(1 \cdot 1)$ \\
Mycobacterium malmoense & $5(0 \cdot 9)$ \\
Mycobacterium kansasii & $2(0 \cdot 4)$ \\
Others & $8(1 \cdot 5)$ \\
\hline \hline
\end{tabular}

NTM, Non-tuberculous mycobacteria.

only one species from two sources and $17(3.7 \%)$ had one species from three different source. Twenty-five patients $(5 \cdot 4 \%)$ had two or three different NTM species isolated from $\geqslant 2$ sources: $21(4 \cdot 6 \%)$ had two species from two sources, three $(0.6 \%)$ had two species from three sources and one $(0 \cdot 2 \%)$ had three species from three sources. The most prevalent NTM species were $M$. xenopi $(219,39 \cdot 5 \%)$, and mycobacteria of the M. avium complex (MAC) (216, $38.9 \%$ ) followed by the Rapidly Growing Mycobacteria group (39, 7\%) (Tables 1 and 2).

\section{Clinical data}

A subset of 88 patients, admitted to the Infectious Disease Unit and the Section of Respiratory Medicine, were included for the analysis of clinical data. According to ATS/IDSA criteria, we classified $15(17 \%)$ patients as NTM-PD cases and $73(83 \%)$ as colonized patients. Of these, 10 patients were considered colonized (although they met clinical, microbiological and radiological criteria), because another 
Table 2. Epidemiological characteristics of the 462 patients from which the NTM were obtained

\begin{tabular}{ll}
\hline \hline Variable & Population, $n$ \\
\hline Male $(\%)$ & $305(66)$ \\
Female $(\%)$ & $157(34)$ \\
Mean age, years ( \pm s.D.) & $64( \pm 18)$ \\
Median age, years & 69 \\
Geographical regions, $n(\%)$ & \\
Europe & $413(89 \cdot 4)$ \\
Italy & 393 \\
Other countries & 20 \\
Eastern Mediterranean & $15(3 \cdot 3)$ \\
South East Asia & $4(0 \cdot 9)$ \\
Western Pacific & $12(2 \cdot 6)$ \\
Africa & $10(2 \cdot 2)$ \\
Americas & $3(0 \cdot 6)$ \\
Not available & $5(1)$ \\
Isolation/isolation sources, $n(\%)$ & \\
$1 / 1$ & $391(84 \cdot 6)$ \\
$1 / 2$ & $29(6 \cdot 3)$ \\
$1 / 3$ & $17(3 \cdot 7)$ \\
$2 / 2$ & $21(4 \cdot 6)$ \\
$2 / 3$ & $3(0 \cdot 6)$ \\
$3 / 3$ & $1(0 \cdot 2)$ \\
\hline \hline
\end{tabular}

NTM, Non-tuberculous mycobacteria.

diagnosis excluded NTM-PD: acute exacerbation of COPD (three patients), tuberculosis (one patient), lung cancer (three patients), pneumonia (one patient), exacerbation of cystic fibrosis (one patient), sarcoidosis (one patient) (Fig. 1). The mean of examined respiratory samples in colonized patients was $3 \cdot 8$.

The baseline characteristics of patients are shown in Table 3. Comparing colonized and NTM-PD patients we did not find statistically significant differences of age, gender and comorbidity between the two groups.

There were more male than female patients colonized by NTM, while NTM-PD appeared to be more prevalent in females.

Comparing the considered symptoms and signs, we found some differences between colonized and NTM-PD patients. However, only fatigue was significantly more prevalent in NTM-PD patients $(P=$ 0.009). Moreover, 14 NTM-PD patients (93.3\%) had chronic or recurrent cough, which was a higher percentage than in colonized patients $(75 \cdot 3 \%)$. Analysing radiological features, NTM-PD patients presented cavitary lesions at a higher percentage than colonized patients $(P=0 \cdot 06)$.

MAC species were prevalent in the 15 NTM-PD patients: $M$. avium and $M$. intracellulare were associated with NTM-PD $(P=0.001)$, whereas $M$. xenopi was statistically associated with colonization $(P=$ $0 \cdot 009)$.

\section{DISCUSSION}

Despite the improvements of radiological and microbiological techniques the diagnosis of NTM disease remains a challenging task, due to the difficulty in ascribing clinical significance of NTM isolates from the respiratory tract [12].

In 2010 Winthrop stated that it was still necessary to clarify some queries about NTM epidemiology and to determine risk factors for NTM-PD [15].

In the past years many laboratories have reported changes in the number and species of NTM isolated from clinical specimens: those isolations changed according to different geographical areas and different species emerged. In European countries increased isolation of M. xenopi has been described [1]. We did not study the incidence of species isolated over the years, but we considered the whole number of NTM species isolated between 2011 and 2012 in our laboratory and the most prevalent species was M. xenopi $(39 \cdot 5 \%)$, followed by MAC species (38.9\%). This finding is in accord with data reported in a recent study by van der Werf et al. [16], which collected data from several national tuberculosis reference laboratories of the European Union, although the authors did not specify the source of NTM isolates. In Italy, in the period 2001-2010, these authors reported 2498 NTM isolates obtained from all types of specimens and the prevalent species encountered was $M$. xenopi $(687,27 \cdot 5 \%)$, followed by $M$. avium (350, 14\%) [16].

In our study we applied ATS/IDSA criteria to distinguish NTM-PD patients from colonized patients and we also tried to identify risk factors for NTM-PD. We found only $17 \%$ of patients satisfying ATS criteria, a lower percentage than in the study of Del Giudice et al., [17], carried out between December 2006 and September 2009. In this Italian retrospective study, Del Giudice et al. identified 39 patients who fulfilled ATS/IDSA microbiological criteria (two positive cultures from the same mycobacteria species sputum or one positive culture from BAL or transbronchial or lung biopsy). Next, they studied these 39 patients for the presence or not of the other ATS/IDSA criteria (respiratory symptoms, exclusion of other diseases, radiological criteria). Sixteen $(41 \%)$ patients fulfilled ATS/IDSA criteria [17]. This percentage is higher than those reported in our study and other European studies, probably due 


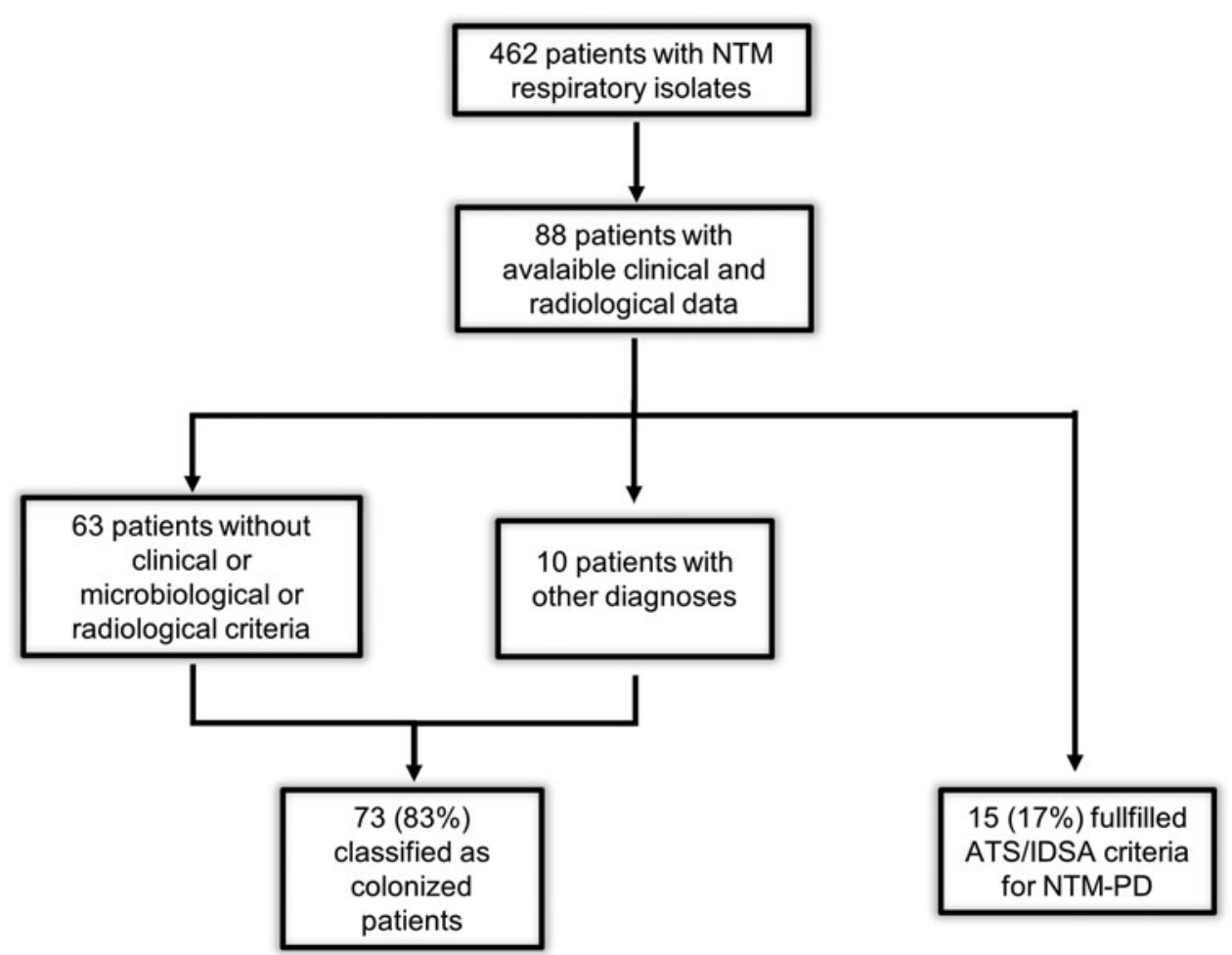

Fig. 1. Flowchart of the study. ATS/IDSA, American Thoracic Society/Infectious Disease Society of America; NTM, non-tuberculous mycobacteria; NTM-PD, non-tuberculous mycobacteria pulmonary disease.

to different enrolment criteria. Indeed, our study and those other studies enrolled patients that only had at least one positive sample and did not necessarily satisfy ATS microbiological criteria. A Greek study considered a period from 2007 to 2013 and described 120 patients with at least one positive culture for respiratory NTM isolation. Fifty-six $(46 \%)$ patients met microbiological criteria, but only $12(16 \%)$ of the patients with adequate clinical data fulfilled the ATS/IDSA criteria [18]. In The Netherlands, van Ingen et al. reviewed retrospectively medical files of patients with positive samples for NTM between January 1999 and January 2005. He found 212 patients with NTM isolates from the respiratory tract of which $53(25 \%)$ met ATS/IDSA criteria [3].

Regarding the symptoms suggestive for NTM-PD, weight loss did not reach the statistically significant association, but the results suggested a trend for a possible association with NTM-PD $(P=0 \cdot 06)$; only fatigue was significantly associated with NTM-PD $(P=0 \cdot 009)$. Van Ingen et al. reported the same association between fatigue and NTM-PD [3]; however, it is a non-specific symptom burdened by a high risk of recall bias.

High-resolution computed tomography can surely contribute to evaluation of the radiological findings typical for NTM-PD: in our study, we observed that cavitary lesions and bronchiectasis are prevalent in NTM-PD (26.6\% and 53.3\%, respectively) compared to colonized patients ( $8 \cdot 2 \%$ and $27 \cdot 4 \%$, respectively), suggesting a trend of possible association, although the low number of patients did not allow a statistically significant association to be obtained. As is known, two radiological patterns are the most frequent, i.e. thin-walled cavities with surrounding parenchymal opacity in the fibrocavitary lung disease; and multifocal bronchiectasis and small nodules in the mid and lower lung field typical of the non-cavitary pattern. Other radiological features could be ground glass pattern, or single nodule, consolidation. However, in the clinical setting, it is very difficult to relate the radiological findings with NTM-PD. It is important to note that other diseases could show these radiological findings: cavitary lesions can occur in pulmonary malignancy, and in infections due to fungi or Nocardia species. Similarly, the bronchiectasis-nodular type pattern was quite specific for NTM-PD, but other diseases could present with similar lesions (cystic fibrosis, primary ciliary dyskinesia, tuberculosis, nocardiosis, and other diseases that cause bronchiectasis).

In our NTM-PD patients, the most prevalent species were $M$. avium (six patients, $40 \%$ ) and $M$. intracellulare 
Table 3. Baseline characteristics of the 88 NTM-infected patients (colonized or NTM-PD) included in the analysis of the clinical data

\begin{tabular}{|c|c|c|c|c|}
\hline Variable & $\begin{array}{l}\text { NTM colonized } \\
(N=73) \\
n(\%)\end{array}$ & $\begin{array}{l}\text { NTM-PD } \\
(N=15) \\
n(\%)\end{array}$ & $P$ & Odds ratio $(95 \% \mathrm{CI})$ \\
\hline Mean age, years ( \pm s.D.) & $60 \cdot 3 \pm 19 \cdot 7$ & $62 \cdot 6 \pm 11 \cdot 6$ & $0 \cdot 91$ & - \\
\hline Median age, years & 65 & 61 & $0 \cdot 92$ & - \\
\hline \multicolumn{5}{|l|}{ Sex } \\
\hline Male & $48(65 \cdot 8)$ & $7(46 \cdot 7)$ & $0 \cdot 16$ & $0 \cdot 46(0 \cdot 13-1 \cdot 64)$ \\
\hline Female & $25(34 \cdot 2)$ & $8(53 \cdot 3)$ & & \\
\hline COPD & $22(30 \cdot 1)$ & $7(46 \cdot 7)$ & $0 \cdot 45$ & $2 \cdot 03(0 \cdot 55-7 \cdot 26)$ \\
\hline HIV & $2(2 \cdot 7)$ & $1(6 \cdot 7)$ & $0 \cdot 43$ & $2 \cdot 54(0 \cdot 04-51 \cdot 18)$ \\
\hline Diabetes mellitus & $10(13 \cdot 7)$ & $3(20)$ & $0 \cdot 69$ & $1 \cdot 57(0 \cdot 24-7 \cdot 42)$ \\
\hline \multicolumn{5}{|l|}{ Species isolated } \\
\hline M. abscessus & $2(2 \cdot 7)$ & 0 & & - \\
\hline M. avium & $4(5 \cdot 5)$ & $6(40)$ & $0 \cdot 001$ & $11 \cdot 5(2 \cdot 15-64 \cdot 11)$ \\
\hline M. chimaera & $20(27 \cdot 4)$ & $2(13 \cdot 3)$ & $0 \cdot 34$ & $0 \cdot 41(0 \cdot 04-2 \cdot 08)$ \\
\hline M. fortuitum & $2(2 \cdot 7)$ & 0 & & - \\
\hline M. gordonae & $5(6 \cdot 8)$ & 0 & & - \\
\hline M. intracellulare & $1(1 \cdot 4)$ & $4(26 \cdot 7)$ & $0 \cdot 001$ & $26 \cdot 18(2 \cdot 18-1305 \cdot 94)$ \\
\hline M. malmoense & 0 & $1(6 \cdot 7)$ & & - \\
\hline M. massiliense & $1(1 \cdot 4)$ & 0 & & - \\
\hline M. xenopi & $38(52 \cdot 1)$ & $2(13 \cdot 3)$ & $0 \cdot 009$ & $0 \cdot 14(0 \cdot 01-0 \cdot 71)$ \\
\hline Positive specimens/patients (mean) & $1 \cdot 5$ & $4 \cdot 9$ & $0 \cdot 001$ & - \\
\hline \multicolumn{5}{|l|}{ Respiratory symptoms } \\
\hline Chronic or recurrent cough & $55(75 \cdot 3)$ & $14(93 \cdot 3)$ & $0 \cdot 19$ & $4 \cdot 58(0 \cdot 6-204 \cdot 3)$ \\
\hline Sputum & $34(46 \cdot 6)$ & $10(66 \cdot 7)$ & $0 \cdot 26$ & $2 \cdot 29(0 \cdot 63-9 \cdot 35)$ \\
\hline Dyspnoea & $35(47 \cdot 9)$ & $5(33 \cdot 3)$ & $0 \cdot 63$ & $0.54(0 \cdot 13-1 \cdot 97)$ \\
\hline Haemoptysis & $16(21 \cdot 9)$ & $3(20)$ & $1 \cdot 00$ & $0 \cdot 89(0 \cdot 14-3 \cdot 9)$ \\
\hline \multicolumn{5}{|l|}{ Constitutional symptoms } \\
\hline Fever & $40(54 \cdot 8)$ & $8(53 \cdot 3)$ & $0 \cdot 86$ & $0 \cdot 94(0 \cdot 27-3 \cdot 41)$ \\
\hline Fatigue & $9(12 \cdot 3)$ & $6(40)$ & $0 \cdot 009$ & $4 \cdot 74(1 \cdot 09-19 \cdot 21)$ \\
\hline Weight loss & $9(12 \cdot 3)$ & $5(33 \cdot 3)$ & $0 \cdot 06$ & $3 \cdot 56(0 \cdot 76-14 \cdot 81)$ \\
\hline Night sweating & 0 & $1(6 \cdot 7)$ & $0 \cdot 40$ & - \\
\hline \multicolumn{5}{|l|}{ Radiographical findings } \\
\hline Nodules & $25(34 \cdot 2)$ & $8(53 \cdot 3)$ & $0 \cdot 13$ & $2 \cdot 19(0 \cdot 61-7 \cdot 95)$ \\
\hline Cavitations & $6(8 \cdot 2)$ & $4(26 \cdot 6)$ & $0 \cdot 06$ & $4 \cdot 06(0 \cdot 71-20 \cdot 17)$ \\
\hline Bronchiectasis & $20(27 \cdot 4)$ & $8(53 \cdot 3)$ & $0 \cdot 06$ & $3 \cdot 03(0 \cdot 83-11 \cdot 11)$ \\
\hline
\end{tabular}

CI, Confidence interval; COPD, Chronic obstructive pulmonary disease; HIV, human immunodeficiency virus; NTM, nontuberculous mycobacteria; NTM-PD, non-tuberculous mycobacteria pulmonary disease; s.D., standard deviation.

(four patients, $26 \cdot 7 \%$ ), and although the number of patients and NTM isolates is low to allow firm conclusions to be made, those two species were significantly associated with NTM-PD $(P=0 \cdot 001)$. In the literature, the association between species and NTM-PD is debated: some studies report that MAC is the most common species isolated in patients with definite disease in North America, Asia-Australia and Europe [19-21]. By contrast, some authors observed that no NTM species predicted pulmonary disease [22]. In our analysis $M$. xenopi was prevalent in colonized patients with only two cases of NTM-PD, this is in contrast with findings of other studies that report this species as the second cause of disease after MAC [23]. Moreover, a systematic review on M. xenopi pulmonary disease showed $45 \%$ of patients with advanced NTM-PD and a high prevalence of males $(80 \%)$, data that we cannot confirm due to the low number of observed cases [24].

With regard to the characteristics of the host, we observed a median age of 65 years in colonized patients and 61 years in NTM-PD patients, without significant difference. Elderly people are more susceptible to NTM-PD and colonization: in other studies the mean age is between 50 and 70 years, without significant difference with colonized patients; as we found 
[25]. A retrospective study carried out in a Canadian population identified risk factors for NTM colonization, comparing NTM-colonized patients with a control group of individuals with culture-negative sputum: by multivariate analysis the predictors of NTM colonization included age $\geqslant 60$ years and female sex [26]. Older age was a risk factor for NTM-PD, but it was not able to distinguish disease from colonization.

NTM-PD occurs prevalently in two groups of patients: white middle-aged or elderly men with risk factors (e.g. underlying lung disease including COPD) and elderly non-smoking women without risk factors (e.g. Lady Windermere syndrome) [27]. In our study only one woman presented the features of Lady Windermere syndrome and four elderly men had underlying lung disease (COPD), of whom three had exposure to tobacco (data not shown). The other patients could not be categorized into either of those two groups.

In contrast to our data [which report no difference of gender within the NTM-PD group (eight women $v s$. seven men)], studies in Oregon reported a prevalence of elderly women with NTM-PD [2, 28]. Conversely, European reports show a prevalence of elderly men affected by NTM-PD [3, 17]. Our finding could be explained by considering the limited number of patients and the women's median age that was higher than men's median age (67 years $v s$. $57 \cdot 6$ years, without significant difference). This difference was consistent with the data reported in Oregon.

In the literature, COPD and other pre-existing pulmonary diseases were risk factors for NTM infection [29]. A high percentage of our patients had a diagnosis of COPD, especially in NTM-PD (43.7\%). Fowler et al investigated the prevalence of NTM in a cohort of patients with bronchiectasis due to several causes (e.g. Cystic fibrosis transmembrane conductance regulator mutation, idiopathic, immune deficiency) and 10/98 patients presented a positive sputum culture for NTM, not necessarily associated with NTM-PD [30]. The prevalence and clinical relevance of NTM isolation in COPD was analysed by Hoefsloot et al. in patients presenting with an acute exacerbation of COPD; NTM were isolated from $22 \%$ of those patients [31]. A Danish study reported an association between COPD and NTM-PD, especially when associated with inhaled corticosteroid use [32]. Thus the association between COPD and NTM is still not clearly defined.

The main limitations of our study are that it is retrospective and the number of patients with
NTM-PD is too low to allow a powerful statistical analysis.

In conclusion, our study confirms the difficulty in discriminating between NTM colonization and NTM-PD using the known risk factors or based on clinical, radiological or microbiological data. Based on the current lack of strong evidence in this field, in clinical practice only the monitoring of colonized patients could allow exclusion of the diagnosis of NTM-PD.

Further prospective studies and new biological markers are needed to identify new tools that can be useful for the diagnosis of NTM-PD, and also to clarify if NTM-colonized patients are at higher risk for the development of pulmonary disease over the course of months or years.

\section{ACKNOWLEDGEMENTS}

This study received no financial support.

\section{DECLARATION OF INTEREST}

None.

\section{REFERENCES}

1. Martin-Casabona N, et al. Non-tuberculous mycobacteria: patterns of isolation. A multi-country retrospective survey. International Journal of Tuberculosis and Lung Disease 2004; 8: 1186-1193.

2. Winthrop KL, et al. Pulmonary nontuberculous mycobacterial disease prevalence and clinical features: an emerging public health disease. American Journal of Respiratory and Critical Care Medicine 2010; 182: 977-982.

3. Van Ingen $\mathbf{J}$, et al. Clinical relevance of non-tuberculous mycobacteria isolated in the Nijmegen-Arnhem region, the Netherlands. Thorax 2009; 64: 502-506.

4. Polverosi R, et al. High resolution CT of nontuberculous mycobacteria pulmonary infection in immunocompetent, non-HIV positive patients. Radiologia Medica 2010; 115: 191-204.

5. Glassroth J. Pulmonary disease due to nontuberculous mycobacteria. Chest 2008; 133: 243-251.

6. Taillard C, et al. Clinical implications of Mycobacterium kansasii species heterogeneity: Swiss National Survey. Journal of Clinical Microbiology 2003; 41: 1240-1244.

7. Koh WJ, et al. Clinical significance of the differentiation between Mycobacterium avium and Mycobacterium intracellulare in $M$. avium complex lung disease. Chest 2012; 142: 1482-1488.

8. Kikuchi T, et al. Association between mycobacterial genotypes and disease progression in Mycobacterium avium pulmonary infection. Thorax 2009; 64: 901-907. 
9. Hoefsloot W, et al. Clinical relevance of Mycobacterium malmoense isolation in the Netherlands. European Respiratory Journal 2009; 34: 926-931.

10. Weiss CH, Glassroth J. Pulmonary disease causes by nontuberculous mycobacteria. Expert Review of Respiratory Medicine 2012; 6: 597-613.

11. Griffith DE, et al. An official ATS/IDSA statement: diagnosis, treatment and prevention of nontuberculous mycobacteria diseases. American Journal of Respiratory and Critical Care Medicine 2007; 175: 367-416.

12. Van Ingen J. Diagnosis of nontuberculous mycobacterial infections. Seminars in Respiratory and Critical Care Medicine 2013; 34: 103-109.

13. Russo C, Tortoli E, Menichella D. Evaluation of new GenoType Mycobacterium assay for identification of mycobacterial species. Journal of Clinical Microbiology 2006; 44: 334-339.

14. Tortoli E, et al. Commercial DNA probes for mycobacteria incorrectly identify a number of less frequently encountered species. Journal of Clinical Microbiology 2010; 48: 307-310.

15. Winthrop KL. Pulmonary disease due to nontuberculous mycobacteria: an epidemiologistic's view. Future Microbiology 2010; 5: 343-345.

16. van der Werf MJ, et al. Inventory study of nontuberculous mycobacteria in the European Union. BMC Infectious Diseases 2014; 14: 62.

17. Del Giudice G, et al. Nontuberculous mycobacterial lung disease in patients without HIV infection: a retrospective analysis over 3 years. Clinical Respiratory Journal 2011; 5: 203-210.

18. Panagiatou M, et al. The epidemiology of pulmonary nontuberculous mycobacteria: data from a general hospital in Athens, Greece, 2007-2013. Pulmonary Medicine. Published: 10 June 2014. doi: 10.1155/2014/ 894976.

19. Koh WJ, et al. Clinical significance of nontuberculous mycobacteria isolated from respiratory specimens in Korea. Chest 2006; 129: 341-348.

20. Kendal BA, Winthrop KL. Update on the epidemiology of pulmonary nontuberculous mycobacterial infections.
Seminars in Respiratory and Critical Care Medicine 2013; 34: 87-94.

21. Simons S, et al. Nontuberculous mycobacteria in respiratory tract infections, Eastern Asia. Emerging Infectious Diseases 2011; 17: 343-349.

22. Bodle EE, et al. Epidemiology of nontuberculous mycobacteria in patients without HIV infection, New York City. Emerging Infectious Diseases 2008; 14: 390-396.

23. Marras TK, et al. Isolation prevalence of pulmonary nontuberculous mycobacteria in Ontario, 1997-2003. Thorax 2007; 62: 661-666.

24. Varadi RG, Marras TK. Pulmonary Mycobacterium xenopi infection in non-HIV-infected patients: a systematic review. International Journal of Tuberculosis and Lung Disease 2009; 13: 1210-1218.

25. Mirsaeidi M, et al. Management on nontuberculous mycobacterial infection in elderly. European Journal of Internal Medicine 2014; 25: 356-363.

26. Hernandez-Garduno E, Elwood RK. Demographic risk factors of pulmonary colonization by non-tuberculous mycobacteria. International Journal of Tuberculosis and Lung Disease 2010; 14: 106-112.

27. Reich JM, Johnson RE. Mycobacterium avium complex pulmonary disease presenting as an isolated lingular or middle lobe pattern. The Lady Windermere syndrome. Chest 1992; 101: 1605-1609.

28. Cassidy PM, et al. Nontuberculous Mycobacterial disease prevalence and risk factors: a changing epidemiology. Clinical Infectious Diseases 2009; 49: e124-129.

29. Piersimoni C, Scarparo C. Pulmonary infections associated with nontuberculous mycobacteria in immunocompetent patients. Lancet Infectious Diseases 2008; 8: 323-334.

30. Fowler SJ, et al. Nontuberculous mycobacteria in bronchiectasis: prevalence and patient characteristics. European Respiratory Journal 2006; 28: 1204-1210.

31. Hoefsloot W, et al. Prevalence of nontuberculous mycobacteria in COPD patients with exacerbations. Journal of Infection 2013; 66: 542-545.

32. Andrejak $\mathbf{C}$, et al. Chronic respiratory disease, inhaled corticosteroids and risk of non-tuberculous mycobacteriosis. Thorax 2013; 68: 256-262. 